\title{
NGHIÊN CỨU PHƯƠNG PHÁP XÁC ĐINNH VANILLIN, ETHYL VANILIIN, MENTHOL, AMYL ACETAT, CITRAL TRONG NỂN MẪU PHỤ GIA THỰC PHẨM BẲNG SẮC KÝ KHÍ KHỐI PHỔ (GC-MS)
}

Lê Việt Ngân'1, Vũ Thị Kim Oanh, Vũ Thị Trang, Lưu Thị Huyền Trang

Viện Kiểm nghiệm an toàn vệ sinh thực phẩm Quốc gia

(Ngày đến tòa soạn: 26/1/2018; Ngày sủa bài sau phản biện: 5/3/2018; Ngày chấp nhận đăng: 14/3/2018)

Tóm tắt

UUC tiêu của nghiên cúu là khảo sát các điều kiện xử lý mẫu và phân tích trên hệ thống sắc ký khí 1 khối phổ để xác định vanillin, ethyl vanillin, menthol, amyl acetat, citral trong nền mẫu phu gia thực phẩm. Các chất phân tích được chiết ra khỏi nền mẫu bằng acetonitril tại $30^{\circ} \mathrm{C}$ trong 15 phút, tách

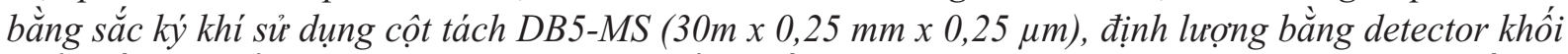
phổ. Kết quả thẩm định thu được đường chuẩn tuyến tính trong khoảng 0,05- $20 \mu \mathrm{g} / \mathrm{mL}$, hiệu suất thu hồi 95,0 - 105\%, độ lạp lại RSD 1,92 - 4,04\%. Úng dụng phwơng pháp phân tích 15 mẫu phu gia thưc phẩm tạo hưong thu thập trên thị trường cho thấy hầu hết các mẫu phu gia huơng liệu chứa nhiều hơn một hơp chất tạo hưong trong thành phần.

Tù khóa: GC-MS, hợp chất tạo hương, phu gia thực phẩm

\section{1. ĐĂT VẤN ĐỀ}

Phụ gia thực phẩm là những chất được sử dụng trong thực phẩm nhằm hỗ trợ hoặc tăng cường chất lượng sản phẩm cuối cùng hoặc trong quá trình chế biến. Các phụ gia thực phẩm giúp tăng thêm một số tính chất cần thiết cho thực phẩm như nâng cao chất lượng và sự ổn định các sản phẩm thực phẩm; duy trì giá trị dinh dưỡng cho thực phẩm; duy trì tính cảm quan của thực phẩm.

Hương liệu là một trong những phụ gia thực phẩm được sử dụng phổ biến để thay đổi và duy trì tính chất cảm quan của sản phẩm. Một loại hương có thể được tạo nên từ một hợp chất hoặc hỗn hợp của nhiều hợp chất khác nhau. Các chất được tìm thấy trong chiết xuất tinh dầu vỏ bưởi: tanal, citronellal, trans-p-mentha-2,8-dien-1-ol, decanal, dodecanal, geranial, cumin aldehyde, nootkatone. Các chất được tìm thấy trong chiết xuất tinh dầu vỏ cam: tanal, citronellal, nonalal, decanal, geranial [1]. Có 43 và 26 hợp chất tương ứng được định lượng trong tinh dầu chuối và chuối tươi xay [6]. Từ các tài liệu tham khảo được và sự hiểu biết về ngành khoa học tạo hương, nhận thấy các chất tạo hương không tồn tại riêng lẻ trong một loại mẫu và một chất tạo hương có thể có mặt trong nhiều loại mẫu khác nhau. Nghiên cứu này phát triển phương pháp phân tích đồng thời vanillin, ethyl vanillin, methol, amyl acetate và citral trong phụ gia thực phẩm hương cam, hương chanh, hương bạc hà, hương chuối, hương vani.

Có nhiều nghiên cứu về phương pháp xác định các hợp chất tạo hương đã được công bố trên thế giới sử dụng các kỹ thuật phân tích cũng rất đa dạng: sắc ký khí, sắc ký lỏng kết hợp với detector quang (UV-Vis, PDA), khối phổ, detector FID, ESI $[2,3,4,5]$. Tuy nhiên do tính chất dễ bay hơi của các hợp chất tạo hương mà việc sử dụng sắc ký khí có được những ưu điểm vượt hơn. Và việc sử dụng detector khối phổ cho phép nhận dạng chính xác chất phân tích.

\section{2. ĐỐI TƯợNG VÀ PHƯƠNG PHÁP NGHIÊN CÚU}

Các chất chuẩn sử dụng trong nghiên cứu: amyl acetate, citral, vanillin, ethyl vanillin, menthol từ Sigma Aldrich; Các dung môi: acetonitril, methanol, n-hexan, diclomethan từ Merck.

Phương pháp phân tích sử dụng máy sắc ký khí khối phổ (GC-MS) (Agilent) với cột sắc ký khí DB5 MS (30m x 0,25mm x $0,20 \mu \mathrm{m})$

Đối tượng mẫu gồm các loại phụ gia thực phẩm: hương chanh, hương cam, hương chuối, hương

${ }^{1}$ Điện thoại:01688288081 Email:nganbmt113@gmail.com 
bạc bà, hương vani được lấy ngẫu nhiên trên địa bàn Hà Nội

3. KẾT QUẢ VÀ BÀN LUẬN

\subsection{Khảo sát các điều kiện phân tích bằng sắc ký khí khối phổ GC-MS}

Cố định các điều kiện phân tích sắc ký khí: tốc độ dòng khí: $1 \mathrm{~mL} /$ phút, thể tích tiêm mẫu: $1 \mu \mathrm{L}$, nhiệt độ tiêm mẫu: $200^{\circ} \mathrm{C}$, dung môi phân tích: acetonitril, cột sắc ký khí DB5-MS $(30 \mathrm{~m}$ x $0,25 \mathrm{~mm}$ $\mathrm{x} 0,25 \mu \mathrm{m})$. Khảo sát điều kiện nhiệt độ ban đầu và gradient nhiệt độ của quá trình phân tích cho thấy: amyl acetat có khối lượng phân tử nhỏ, mạch cấu tạo gọn, dễ bay hơi, do vậy cần nhiệt độ ban đầu thấp để kéo dài thời gian lưu giữ trong cột, tách được với các dung môi và tạp chất không lưu giữ khác. Vanillin và ethyl vanillin có khả năng lưu giữ tốt, thời gian lưu dài khiến pic có độ đối xứng kém, kéo đuôi lớn, do vậy cần tăng nhiệt độ để hai chất này được rửa giải nhanh hơn. Kết quả chương trình gradient tối ưu được đưa ra trong bảng 1 .

Bảng 1. Chương trình nhiệt độ buồng cột

\begin{tabular}{|c|c|c|c|}
\hline & Tốc độ tăng ( $\left.{ }^{o} \mathrm{C} / p h u ́ t\right)$ & Nhiệt độ $\left({ }^{\circ} C\right)$ & Thòi gian giũu (phút) \\
\hline (Ban đầu) & & 50 & 1 \\
\hline Chu kỳ 1 & 15 & 100 & 1 \\
\hline Chu kỳ 2 & 50 & 280 & 5 \\
\hline
\end{tabular}

\subsection{Khảo sát quy trình xử lý mẫu}

\subsubsection{Khảo sát dung môi chiết mẫu}

Thực hiện khảo sát khả năng hòa tan của các chất phân tích trong các dung môi khác nhau cho thấy: khi sử dụng etanol, methanol, acetonitril mẫu được hòa tan nhanh, tạo dung dịch đồng nhất; khi sử dụng $n$-hexan mẫu chỉ tan một phần và có hiện tượng phân lớp không đồng nhất. Dựa trên loại dung môi thích hợp nhất đối với phân tích sắc ký khí, acetonitril đã được lựa chọn trong nghiên cứu này làm dung môi chiết và hòa tan chất phân tích 3.2.2 Khảo sát nhiệt độ và thời gian chiết

Thực hiện khảo sát chiết mẫu ở các nhiệt độ $30^{\circ} \mathrm{C}, 40^{\circ} \mathrm{C}, 60^{\circ} \mathrm{C}$ và $80^{\circ} \mathrm{C}$; thời gian chiết 15,30 và 60 phút. Thực hiện phân tích trên hệ thống GC-MS, xác định diện tích peak chất phân tích tương ứng với điều kiện chiết mẫu. Kết quả thể hiện trong hình 1 .

Từ kết quả so sánh cho thấy không có sự khác nhau đáng kể về hàm lượng chất phân tích xác định được tại các điều kiện nhiệt độ và thời gian khác nhau. Do đó, để giảm thời gian phân tích và tiết kiệm chi phí nguyên vật liệu năng lượng, thời gian chiết 15 phút và nhiệt độ chiết $30^{\circ} \mathrm{C}$ được lựa chọn cho các nghiên cứu tiếp theo.

\subsection{Thẩm định phương pháp}

Độ đặc hiệu của phương pháp được đánh giá thông qua việc phân tích mẫu trắng, mẫu chuẩn và mẫu thêm chuẩn. Kết quả chỉ ra mẫu trắng không cho tín hiệu chất phân tích, mẫu chuẩn và mẫu thêm chuân cho tín hiệu chất phân tích tại cùng thời gian lưu, kết quả cho thấy phương

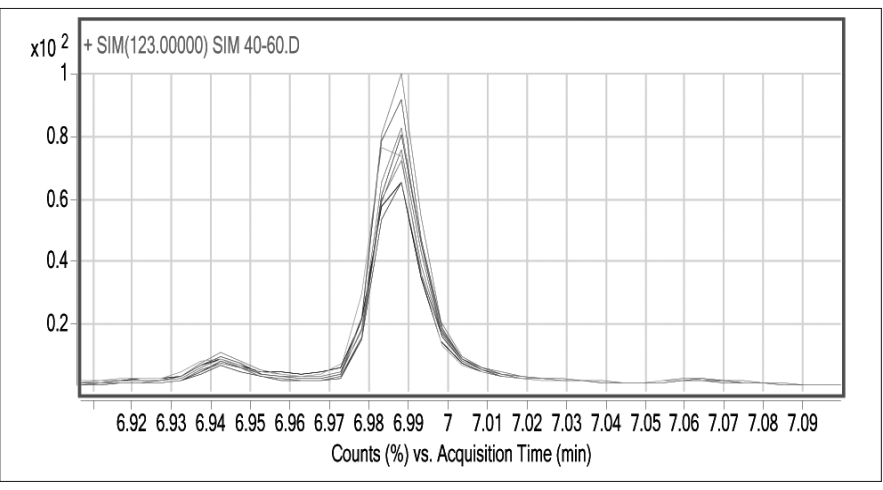

Hình 1. Sắc đồ chồng phổ phân tích menthol trong các điều kiện chiết khác nhau

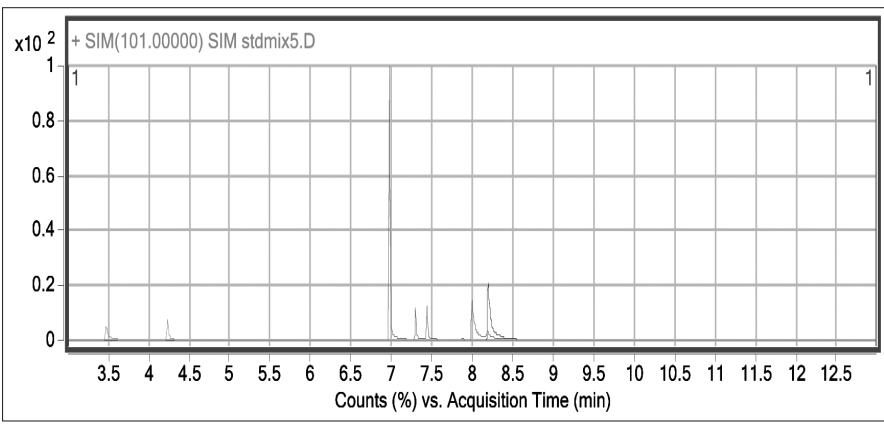

Hình 2. Sắc đồ chuẩn hỗn hợp amyl acetat, citral, menthol, vanillin và ethyl vanillin 
pháp có độ đặc hiệu tốt.

Đường chuẩn phân tích 5 hợp chất tạo hương được lựa chọn đã được thiết lập sử dụng điều kiện phân tích tối ưu, trong khoảng nồng độ $0,05-20 \mu \mathrm{g} / \mathrm{mL}$. Kết quả phương trình đường chuẩn, hệ số tương quan, giới hạn phát hiện (LOD) và giới hạn định lượng (LOQ), độ lặp lại (RSD) và độ thu hồi (R) của các chất phân tích được trình bày trong bảng 2 .

Bảng 2. Kết quả đánh giá phưong pháp phân tích

\begin{tabular}{|c|c|c|c|c|c|c|}
\hline Tên chất & $\begin{array}{l}\text { Phuơng trình } \\
\text { đừ̀ng chuẩn }\end{array}$ & $\begin{array}{c}\text { Hệ số tuơng quan } \\
\qquad R^{2}\end{array}$ & $\begin{array}{c}L O D \\
(\mu g / g)\end{array}$ & $\begin{array}{c}L O Q \\
(\mu g / g)\end{array}$ & $R S D(\%)$ & $R(\%)$ \\
\hline Vanillin & $Y=204944 X-7259$ & 0,9992 & 4 & 13,2 & 4,04 & 93-101 \\
\hline Ethyl vanillin & $Y=18879 X-967$ & 0,9999 & 4 & 13,2 & 3,63 & $95-103$ \\
\hline Menthol & $Y=34828 X-3036$ & 0,9998 & 4 & 13,2 & 1,92 & 96-99 \\
\hline Amyl acetate & $Y=3776 X-80,5$ & 1,0000 & 10 & 33 & 3,49 & $95-104$ \\
\hline Citral & $Y=7999 X+20,3$ & 0,9994 & 10 & 33 & 3,09 & $96-105$ \\
\hline
\end{tabular}

Bảng kết quả tổng hợp thông số thẩm định cho thấy: Hệ số xác định $\mathrm{R}^{2}>0,99$ đối với tất cả các chất phân tích được lựa chọn, độ lặp lại trong khoảng 1,92 đến 4,04\% và độ thu hồi đạt 93\% đến 105\%. Độ lặp lại và độ thu hồi đều đạt yêu cầu theo AOAC. Phương pháp đủ điều kiện để ứng dụng cho phân tích mẫu thực tế.

\section{4. Đánh giá hàm lượng hương liệu trong một số mẫu thực tế}

Tiến hành xác định hàm lượng các chất tạo hương trên 15 mẫu (3 mẫu hương vani, 3 mẫu hương cam, 3 mẫu hương chuối, 3 mẫu hương chanh và 3 mẫu hương bạc hà). Kết quả phân tích được thể hiện trong bảng 3 .

Bảng 3. Kết quả phân tích mẫu thực tế

\begin{tabular}{|c|c|c|c|c|c|}
\hline Tên mâu & $\begin{array}{c}\text { Ethyl vanillin } \\
(\mathbf{m g} / \mathbf{k g})\end{array}$ & $\begin{array}{c}\text { Vanillin } \\
(\mathbf{m g} / \mathbf{k g})\end{array}$ & $\begin{array}{c}\text { Menthol } \\
(\mathbf{m g} / \mathbf{k g})\end{array}$ & Citral (mg/kg) & $\begin{array}{c}\text { Amyl acetat } \\
(\mathbf{m g} / \mathbf{k g})\end{array}$ \\
\hline M1 & 128 & - & - & 1253 & - \\
\hline M2 & - & - & 1889 & - & - \\
\hline M3 & 383 & 1573 & - & - & - \\
\hline M4 & - & 357 & - & 459 & - \\
\hline M5 & - & - & 632 & - & - \\
\hline M6 & 5376 & - & 84 & - & - \\
\hline M7 & - & 1031 & - & - & - \\
\hline M8 & - & - & 861 & 371 & - \\
\hline M9 & - & 1044 & - & - & - \\
\hline M10 & - & 180 & - & - & - \\
\hline M11 & - & - & 350 & 388 & - \\
\hline M12 & - & - & - & 556 & 126 \\
\hline M13 & 352 & - & - & - & - \\
\hline M14 & - & - & - & 165 & - \\
\hline M15 & - & 1506 & - & - & - \\
\hline
\end{tabular}

(-) là nhỏ hơn ngương phát hiện của phương pháp

Kết quả phân tích mẫu thực tế cho thấy các mẫu phụ gia hương liệu đều có thành phần đặc trưng như amyl acetate có trong hương chuối, vanillin và ethyl vanillin có trong hương vani... Tuy nhiên, một phụ gia hương liệu cụ thể lại có nhiều hơn một chất tạo hương trong thành phần như menthol và ethyl 
vanillin xác định được trong hương menthol. Kết quả này một phần là cơ sở để xem xét trong quá trình xây dựng các quy định kỹ thuật về phụ gia thực phẩm hương liệu và quy định hàm lượng của hương liệu trong thực phẩm.

\section{KẾT LUẬN}

Phương pháp xác định vanillin, ethyl vanillin, menthol, amyl acetat, citral trong nền mẫu phụ gia thực phẩm bằng GC-MS đã được xây dựng trên cơ sở tối ưu quy trình chiết mẫu sử dụng acetonitril tại $30^{\circ} \mathrm{C}$ trong 15 phút và đã được xây dựng được quy trình phân tích bằng GC-MS sử dụng cột sắc ký khí

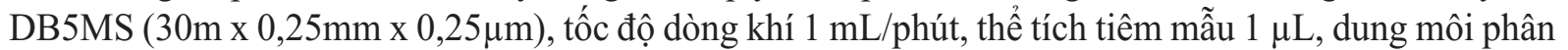
tích: acetonitril với chương trình nhiệt độ thích hợp. Phương pháp được thẩm định có các thông số về độ đặc hiệu, khoảng tuyến tính và đường chuẩn, giới hạn phát hiện, giới hạn định lượng, độ lặp lại và độ thu hồi đều đạt theo quy định AOAC. Phương pháp đã được ứng dụng để phân tích một số mẫu thực tế, xác định được hàm lượng các chất phân tích trong nền mẫu thực tế được thu thập ngẫu nhiên trên địa bàn Hà Nội, kết quả phân tích ban đầu cho thấy đã xác định được một hoặc đồng thời các chất tạo hương đặc trưng cho sản phẩm tương ứng. Kết quả nghiên cứu cho thấy phương pháp đơn giản, có độ tin cậy cao, có khả năng áp dụng tại các phòng thí nghiệm có hệ thống GC-MS.

\section{TÀI LIẸU THAM KHẢO}

1. Hoàng Đình Hòa, Nguyễn Văn Lợi, Nguyễn Thị Minh Tú, "Nghiên cứu thành phần hóa học và hoạt tính sinh học của tinh dầu lá bưởi, cam và chanh", Tạp chí Khoa học và Công nghệ 2014, số 52 (5A), 1 - 6

2. Nguyễn Thị Huyền, Trần Thị Phương Chi "Các chất dễ bay hơi từ loài sả chanh (Cymbopogon citratus) ở Nghệ An”, Hội nghị khoa học toàn quốc về sinh thái và tài nguyên sinh vật lần thứ 6

3. A.G.Huesgen, analysis of natural and artificial vanilla preparations, Agilent Technologies.

4. Diogo Miron et al, "Spectrophotometric simultaneous determination of citral isomers in cyclodextrin com plexs with partial least squares supported approach", Current pharmaceutical analysis, 2012, 8, 401-408

5. Jan Krek, Janusz Slawomir czekaj and Wlodzimierz Rzeszutko "Validation of a method for simulraneuos determination of menthol and methyl salicylate in pharmaceuticals by capillary gas chromatography with cool on-column injection”, Acta Poloniac pharmaceutica Drug Research Vol 60, No.5, pp 343-349, 2003.

6. Maria J.Jordan, Kawaljit Tandon, Philip E. Shaw and Kevin L. Goodner, “Aromatic profile of aqueous ba nana essence and banana fruit by gas chromatography - mass spectrometry (GC-MS) and gas chro matography - Olfactometry (GC-O)", J. Agric. Food Chem. 2001, 49, 4813-4817

\section{Summary}

SIMULTANEOUS DETERMINATION OF VANILLIN, ETHYL VANILLIN, MENTHOL, AMYL ACETATE, CITRAL IN FOOD ADDITIVES BY GAS CHROMATOGRAPHY TAMDEM MASS SPECTROMETRY (GC/MS)

\section{Le Viet Ngan, Vu Thi Kim Oanh, Vu Thi Trang, Luu Thi Huyen Trang \\ National Institute for Food Control}

The objective of this study to determine sample preparation and analysis vanillin, ethyl vanillin, menthol, amyl acetate and citral in the food additives using gas chromatography mass tandem spectrometry. The substances were extracted from the matrices with acetonitrile at $30^{\circ} \mathrm{C}$ for 15 minutes, determined by GC-MS using DB5-MS column ( $30 \mathrm{~m} \times 0.25 \mathrm{~mm} \times 0.25 \mu \mathrm{m})$ for separation, the MS/MS detector. The calibration curves had concentration of 0.05 to $20 \mu \mathrm{g} / \mathrm{mL}$, the method had the recovery of from $95.0 \%$ to $105 \%$, the repeatability of from 1.92 to 4.04\%. Applying the analytical method on 15 flavor food additive samples which were collected from markets showed most of the flavor additives contain more than one flavor compound in composition.

Keywords: GC-MS, aromatic compounds, food additives 